\title{
DETECTABILITY OF BULGE STARS *
}

\author{
M.HERNANDEZ-PAJARES, R.CUBARSI and J.SANZ-SUBIRANA \\ Departament de Matemàtica Aplicada i Telemàtica \\ and \\ J.M.JUAN-ZORNOZA \\ Departament de Física Aplicada \\ ETSETB, Universitat Politècnica de Catalunya, \\ P.O.Box 30002, E-08080 Barcelona, Spain \\ First author's e-mail: matmhp@mat.upc.es
}

\begin{abstract}
Classification algorithms based on neural network techniques are applied to study if the bulge stars can be differentiated from other stars belonging to other Galactic components. A synthetic sample is build as a mixture of four components, thin disk, thick disk, halo and bulge, according to some stellar system models and considering observational errors.
\end{abstract}

Key words: Galactic Bulge - Stellar Classification - Neural Networks

In the recent literature some authors consider directly or indirectly, the possible existence of bulge stars in the solar vicinity. Rich 92 suggests that a central bar might scatter bulge stars, thus providing an explanation for the high velocity metal rich stars in the solar neighbourhood. By other hand Feast et al. 92 propose the existence of a generalized thick disk component, which could be regarded as the Local Bulge type population. This idea is suggested from the study of Mira variables and M-giants local stars. These considerations lead to the problem of the detectability of possible local bulge stars: Which star attributes are necessaries to know, to separate the different components in an efficient way? Can the bulge be detected within a local sample? Which is the possible predominant population that could hide a bulge component? To give first answers to the last questions we follow a synthetic approach within two steeps:

First we generate a four component local sample, with thin disk ( $t$ ), thick disk (T), halo (h) and bulge (b) stars. We suppose, as Nemec et al. 92 do, that the sample has a kinematic bias that allows a strong presence of spheroidal component stars; for instance a proportion of $t: T: h=2: 2: 1$. We introduce a small contamination of bulge stars in this composition with a sample that contains 1000 stars with 400 belonging to each disk component, 150 to the halo and 50 stars to the bulge. The real heliocentric distances have been taken lesser than $2.5 \mathrm{kpc}$ with heights above the galactic plane lesser than $0.5 \mathrm{kpc}$. The sample contains 1000 stars and 8 attributes: 3 -D positions $(r, l, b), 3-\mathrm{D}$ velocities $(U, V, W)$, metallicities $([\mathrm{Fe} / \mathrm{H}])$ and ages. The details with the different distributions adopted for the thin disk, thick disk and halo are explained in Hernández et al. 92. The main features of the local bulge are based in the model of Cubarsi et al. 92 (see Hernández 92).

Secondly, the synthetic catalogue is analyzed with Principal Component Analysis (PCA) and with the Self Organizing Map algorithm (SOM). PCA is a known multivariate technique (see for instance Murtagh et al. 87) that looks for the maximum variance directions and gives us a quantitative idea of which attributes are more discriminant for the sample: in this case the circular rotation component, $V$,

* This work has been supported by the D.G.I.C.I.T. of Spain under Grant No. PB90-0478 


\begin{tabular}{|r|r|r|r|}
\hline 377 & 5 & 0 & 0 \\
\hline 23 & 347 & 9 & 13 \\
\hline 0 & 19 & 139 & 0 \\
\hline 0 & 29 & 2 & 37 \\
\hline
\end{tabular}

TABLE I

The confusion matrix between the thin disk (1), thick disk (2), halo (3) and bulge (4) is given. The number in the $i-t h$ row and $j$-th column indicates the stars that really belong to the population $j$ and appear in a centroid with predominant component $i$.

the metallicity and the age with the $24 \%$ of the total variance. By other hand SOM is an unsupervised neural classifier (see for details Kohonen 90) that has been applied to astronomical data in Hernández et al. 91. It is an efficient way to arrange the stars of the sample in subgroups in terms of the proximity in the characteristic space formed by the $m$-attributes considered, in our case $m=8$. The basic aim is finding a smaller set of centroids that provides a good approximation of the original set $S$ of $n$ stars with $m$ attributes, encoded as "vectors" $x \in S$. However, the main advantage of the algorithm is that it also arranges the centroids so that the associated mapping from $S$ to A maps the topology of the set $S$ in a least distorting way. Usually $\mathbf{A}$ is a bidimensional set of indexes where proximity between them means similarity between the global properties of the associated groups of stars. The SOM of the sample, after 500000 iterations and with a size of $5 \times 5=25$ centroids, has been calculated, and a good discrimination has been obtained by this neural classifier. The bulge is concentrated in two centroids where appears mixed with thick disk stars. Practically all centroids contain a predominant population with more than $50 \%$ of the respective stars. The purity of the classification can be also appreciated looking at the confusion matrix given in Table I. From this table the recognition error is 100 stars $=10 \%$. It is important to note that the main source of confusion happens between the bulge and the thick disk. This component could hide the possible local bulge in observational samples with few bulge stars. Such result is compatible with the identification of local bulge stars with a generalized thick disk component (Feast et al. 92). By other hand, no confusion appears between the thin disk, and the halo and bulge components.

\section{References}

R. Cubarsí and M. Hernández-Pajares, IAU Symp. 153 on Galactic Bulges, 1992.

M.W. Feast, P.A. Whitelock, and R. Sarples, The Stellar Populations of Galaxies, Barbuy and Renzini, eds., IAU Symp. 149, Kluwer Acad. Press, Dordrecht, 1992, p. 77.

M. Hernández-Pajares, to be published, 1992.

M. Hernández-Pajares, R. Cubarsí, and E. Monte, in Heck and Murtagh, Eds., Astronomy from Large Databases II, Eur. Southern Obs., 1992 (proc. , Haguenau, France, 14-16 Sept. 1992).

M. Hernández-Pajares and E. Monte, Artificial Neural Networks, Lecture Notes in Computer Science, vol. 540, Springer-Verlag, Berlin, 1991, p. 422.

T. Kohonen, Proceedings of the IEEE 78 (1990), no. 9, 1464.

F. Murtagh and A. Heck, Multivariate data analysis, D.Reidel Publishing Comp., Dordrecht, 1987.

J.M. Nemec and A.F.L. Nemec, The Stellar Populations of Galaxies, Barbuy and Renzini, eds., IAU Symp. 149, Kluwer Acad. Press, Dordrecht, 1992, p. 103.

R.M. Rich, The Stellar Populations of Galaxies, Barbuy and Renzini, eds., IAU Symp. 149, Kluwer Acad. Press, Dordrecht, 1992, p. 29. 